\title{
REVIEW
}

\section{Everolimus in the Treatment of Renal Cell Carcinoma and Neuroendocrine Tumors}

\author{
Hiu-yan Chan · Ashley B. Grossman · Ronald M. Bukowski
}

Received: April 30, 2010 / Published online: July 8, 2010

(C) The Author(s) 2010. This article is published with open access at Springerlink.com

\section{ABSTRACT}

Renal cell carcinoma (RCC) and neuroendocrine tumors (NET) are uncommon malignancies, highly resistant to chemotherapy, that have emerged as attractive platforms for evaluating novel targeted regimens. Everolimus is an oral rapamycin derivative within the mammalian target of rapamycin class of agents. Preclinical series have shown that everolimus exhibits anticancer effects in RCC and NET cell lines. A phase 3 placebo-controlled study in advanced clear-cell RCC, known as RECORD-1 (for "REnal Cell cancer treatment with Oral RAD001 given Daily"), documented that everolimus stabilizes tumor progression, prolongs progressionfree survival and has acceptable tolerability in patients previously treated with the multikinase inhibitors sunitinib and/or sorafenib. Everolimus has been granted regulatory approval for use in sunitinib-pretreated and/or sorafenib-pretreated

Hiu-yan Chan · Ashley B. Grossman

Barts and the London School of Medicine, London, UK.

Ronald M. Bukowski $(\square)$

Cleveland Clinic Taussig Cancer Center, Cleveland, Ohio, USA. Email: bukow464@sbcglobal.net advanced RCC and incorporated into clinical practice guidelines, and the RECORD- 1 safety data are being used to develop recommendations for managing clinically important adverse events in everolimus-treated patients. Ongoing clinical trials are evaluating everolimus as earlier RCC therapy (first-line for advanced disease and as neoadjuvant therapy), in non-clear-cell tumors, and in combination with various other approved or investigational targeted therapies for RCC. Regarding advanced NET, recently published phase 2 data support the ability of everolimus to improve disease control in patients with advanced NET as monotherapy or in combination with somatostatin analogue therapy, octreotide longacting release (LAR). Forthcoming data from phase 3 placebo-controlled trials of everolimus, one focused on monotherapy for pancreatic NET and the other on combination use with octreotide LAR for patients with advanced NET and a history of carcinoid syndrome, will provide insight into its future place in NET therapy. The results of a number of ongoing phase 3 evaluations of everolimus will determine its broader applicability in treating breast cancer (in combination with chemotherapy and hormonal therapy), several advanced gastrointestinal cancers, hepatocellular carcinoma, and 
lymphoma (in the adjuvant setting), as well as the various lesions associated with the tuberous sclerosis complex tumor suppressor gene.

Keywords: endocrine tumors; everolimus; gastroenteropancreatic; islet cell carcinoma; kidney cancer; mTOR inhibitor; neuroendocrine tumors; RAD001; rapamycin; renal cell carcinoma

\section{INTRODUCTION}

Rapamycin (also known as sirolimus), isolated in soil from the island of Rapa Nui (Easter Island) in the South Pacific in 1975, ${ }^{1}$ was initially recognized as an antifungal and immunosuppressive agent. Its anticancer properties were discovered during the 1980s, at a time when development of rapamycin was focused on the area of antirejection in organ transplantation recipients (resulting in United States and European approval for this use during 1999-2000). The therapeutic potential of rapamycin against a wide range of malignancies, as shown by broad activity in the National Cancer Institute human cancer cell lines, ${ }^{2}$ ultimately fueled the clinical development of a series of derivatives with more favorable pharmacologic and pharmacokinetic profiles; ie, temsirolimus (CCI-779), everolimus (RAD001), and ridaforolimus (AP23573/ MK-8669, formerly deforolimus).

Rapamycin and its derivatives comprise a new class of anticancer agents that block the mammalian target of rapamycin (mTOR) pathway, as shown in Figure 1. mTOR, a serinethreonine kinase, acts as a biochemical switch, ensuring that supplies of energy and nutrients in the cell are sufficient to maintain cell viability and stimulate cell growth, cell division, and angiogenesis. mTOR integrates the signals to affect multiple downstream processes, signaling downstream of key receptor tyrosine kinases such as insulin-like growth factor-1, endothelial growth factor, and vascular endothelial growth factor (VEGF) receptors, among others. Signaling through the phosphatidylinositol-3-kinase (PI3K)/Akt system stimulates its downstream proteins, including mTOR and ultimately p70S6K (the serine/threonine kinase of p70S6). In cancer, this aberrant activation of the mTOR pathway shifts cells into a trajectory favoring cell proliferation and survival, cell growth, and increased cell motility and angiogenesis, considered hallmarks of malignancy.,4 The mTOR inhibitors are termed "downstream multisignal inhibitors", differentiating this class from potential therapeutic agents that act upstream of mTOR. By blocking the mTOR pathway, the Akt-mediated nutrient flux essential for cancer growth and the Akt-mediated antiapoptotic

Figure 1. Growth-stimulating signals originating from within and outside the cell are integrated through mammalian target of rapamycin (mTOR) into processes that maintain cell viability, and stimulate cell growth, cell division, and angiogenesis.

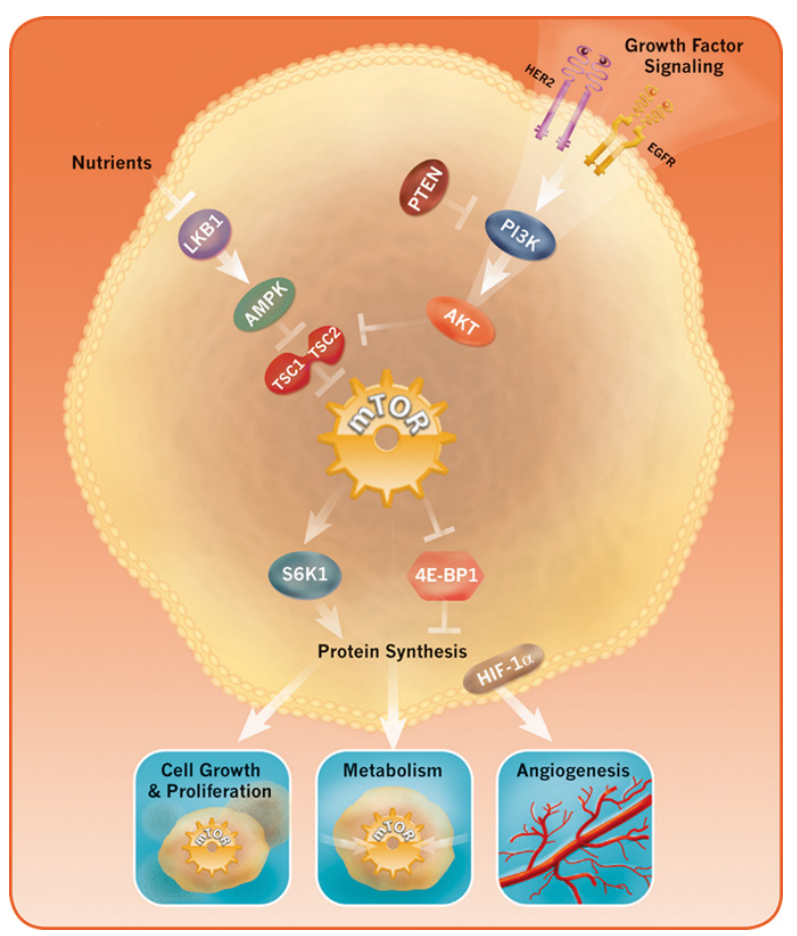


effect are inhibited, attenuating cell growth and slowing proliferation. ${ }^{5}$

Historically, patients with relatively uncommon cancers-including renal cell carcinoma (RCC) and neuroendocrine tumors (NET) - have had few therapeutic options for advanced disease, and in view of the limitations of traditional chemotherapy in these settings, novel molecular therapies were explored. This review will focus on describing clinical experiences to date with the oral rapamycin derivative everolimus in advanced RCC (a recently approved indication) and NET (for which phase 3 data are forthcoming), followed by an overview of ongoing and future directions for the development of this agent for the treatment of cancer.

\section{USE OF EVEROLIMUS IN RCC}

\section{Overview of the RCC Therapeutic Landscape}

RCC, arising from the cells of the proximal tubule, has long been regarded as one of the malignancies with the poorest prognosis, reflecting its characteristic diagnosis at an advanced, unresectable or metastatic stage and inherent resistance to chemotherapy. Surgery, albeit recommended across the various stages of RCC, is met with limited success particularly in advanced disease. Cytokine-based immunotherapy (interferon, interleukin-2) was regarded as standard systemic therapy for advanced RCC for many years ${ }^{6}$ despite producing modest benefit (at best) in conjunction with a high degree of toxicity, with average median survival limited to only 13 months. ${ }^{7}$ The ability of molecular targeted agents-including the VEGF receptor-tyrosine kinase inhibitors (VEGFrTKIs) sunitinib, ${ }^{8,9}$ sorafenib, ${ }^{10}$ and most recently pazopanib, ${ }^{11}$ the anti-VEGF monoclonal antibody bevacizumab (administered in combination with interferon alfa), ${ }^{12}$ and the mTOR inhibitors temsirolimus ${ }^{13}$ and everolimus ${ }^{14,15}$ - to improve outcomes with relatively good tolerability has rapidly revolutionized the treatment of advanced RCC. The clinical benefit of these agents has been mainly in the form of disease stabilization and prolonged progressionfree survival (PFS). A statistically significant overall survival (OS) benefit was demonstrated with temsirolimus relative to interferon alfa in primary analyses. ${ }^{13}$ Secondary analyses, censoring for post-trial anticancer therapies or crossover bias, have demonstrated a significant OS benefit with sunitinib, with a median OS twice that of interferon alfa, ${ }^{9}$ sorafenib, ${ }^{16}$ and everolimus. ${ }^{15,17}$ Although targeted therapies have become the new cornerstone of systemic RCC therapy, eventual treatment-emergent resistance has emerged as a major impediment to long-term success with these new agents and is fueling research efforts to identify optimal sequencing and combinations.

\section{Preclinical/Mechanistic Rationale for Targeting mTOR}

RCC is a highly vascularized malignancy and therefore was identified as a compelling target for antiangiogenesis-based therapy. Most clearcell tumors, the most prominent histologic subtype of RCC (approximately $85 \%$ of cases), are associated with inactivation of the von Hippel-Lindau (VHL) tumor suppressor gene. ${ }^{18}$ VHL-deficient cells are characterized by an upregulation of hypoxia-inducible factor (HIF) and a corresponding increase in VEGF and various other growth and angiogenic factors. ${ }^{18,19}$ mTOR functions as a regulator of HIF and has been shown to be activated in clear-cell RCC, 20,21 as well as in RCC-predisposing tumor suppressor gene syndromes involving tuberous sclerosis complex 1 and 2 (TSC-1/2) and phosphatase 
and tensin homolog (PTEN). ${ }^{19}$ Pantuck et al. examined expression of components of the mTOR pathway in samples from 375 patients who underwent nephrectomy for sporadic RCC, demonstrating particularly high mTOR activation among clear-cell, high-grade, and other poor prognosis tumors. ${ }^{20}$

Preclinically, rapamycin has demonstrated the ability to inhibit the cellular growth of RCC. ${ }^{21}$ More recently, an in vitro series found that everolimus significantly inhibits the growth of RCC cell lines and cell cycle progression (reducing S-phase while increasing G[0]/G[1] cells and altering regulatory protein expression), with an enhancement of antiproliferative and cell cycle attenuating effects when combined with an investigational multikinase inhibitor (AEE788).22

\section{Published Clinical Trials of the Efficacy and Safety of Everolimus in RCC}

The first published phase 2 trial of everolimus (dosed at $10 \mathrm{mg} /$ day) for metastatic RCC, conducted by Amato et al., enrolled 41 patients with predominantly clear-cell disease who had received up to one prior systemic treatment. ${ }^{23}$ Most patients (83\%) had been previously treated, mainly in the form of immunotherapy $(61 \%) .{ }^{23}$ With $57 \%$ of patients progression free for $\geq 6$ months and median PFS of 11.2 months (95\% CI: 1.7, 36.2 months), the study met the prespecified criteria for further evaluation. ${ }^{23}$ In all, 24 of 37 evaluable patients experienced some degree of tumor reduction. ${ }^{23}$ Objective responses per independent assessment were mainly stable disease (SD), lasting for $\geq 3$ and $\geq 6$ months in $74 \%$ and $58 \%$ of patients, respectively, with an additional two patients achieving a partial response (PR). ${ }^{23}$ Considering all patients, median OS was 22.1 months (95\% CI: 1.4, 36.4 months). ${ }^{23}$ Most adverse events (AEs) were of grade $1 / 2$ severity, with no grade 4 AEs reported..$^{23}$ The most common treatment-related grade 3 AEs were pneumonitis $(n=7,18 \%)$ and alanine aminotransferase elevation $(n=4,10.3 \%)$, followed by alkaline phosphatase elevation, hyperglycemia, and thrombocytopenia ( $n=3$ for each, $8 \%){ }^{23}$

RECORD-1 (for "REnal Cell cancer treatment with Oral RAD001 given Daily") was a phase 3 placebo-controlled trial of everolimus $10 \mathrm{mg}$ /day in patients with metastatic RCC progressing after sorafenib and/or sunitinib. Prior therapy with cytokines and/or VEGF inhibitors (eg, bevacizumab) also was permitted. A significant PFS benefit for everolimus at the time of the second interim analysis prompted an early termination of this study. ${ }^{14}$ Based on the interim results derived from independent central review of 410 patients, everolimus was associated with a significant $70 \%$ improvement in PFS, with a median of 4.0 months versus 1.9 months with placebo $(P<0.0001)$ and 6-month PFS rates of $26 \%$ versus $2 \%$, respectively. ${ }^{14}$ PRs were seen in three everolimus recipients and no placebo recipients, but the biggest difference was with respect to a near doubling of the SD rate: $63 \%$ with everolimus versus $32 \%$ with placebo. ${ }^{14}$ The nonsignificant difference in median OS between the two groups, not reached with everolimus versus 8.8 months with placebo (hazard ratio [HR], 0.83; 95\% CI: 0.50, 1.37; $P=0.23)$, was likely confounded by the high rate of crossover from placebo to everolimus at disease progression. ${ }^{14}$ Two valid and independent statistical methods were used to calculate OS for crossover and estimate true survival benefit of everolimus in patients with metastatic RCC. Using a rankpreserving structural failure time (RPSFT) model to correct the treatment effect estimate for crossover bias, estimated survival time was 1.9fold longer (95\% CI: 0.5, 8.5) with everolimus versus placebo. ${ }^{15}$ The RPSFT approach was 
also used to reconstruct the placebo survival curve resulting in a median OS for placebo of 10.0 months. ${ }^{15}$ A second statistical analysis (inverse probability of censoring weights) used to correct for crossover demonstrated that everolimus reduced the risk of death by $45 \%$ (HR, 0.55; 95\% CI: 0.31, 0.97; $P=0.0389) .{ }^{17}$ Updated efficacy based on the final data analysis $(n=416)$ is consistent with the interim results, with median PFS of 4.9 months with everolimus and 1.9 months with placebo (HR, $0.33 ; P<0.001)$ and corresponding median OS of 14.8 months and 14.4 months (HR, $0.87 ; P=0.18) .{ }^{15}$ Regarding the final safety data for everolimus, stomatitis (44\%) and infections (37\%) were the most common AEs, more common than with placebo ( $8 \%$ and $18 \%$, respectively) but typically of grade $1 / 2$ severity. ${ }^{15}$ Grade $3 / 4$ AEs in $\geq 5 \%$ of everolimus recipients were infections (10\%), dyspnea (7\%), and fatigue (5\%). ${ }^{15}$ Noninfectious pneumonitis was exclusively seen in the everolimus arm, reported in 37 patients or $14 \%$ ( $n=9$ grade 1 ; $n=18$ grade $2 ; n=10$ grade 3$).{ }^{15}$ Laboratory abnormalities were also more common with everolimus, predominantly hemoglobin (92\%) and lymphocyte (51\%) reductions and increases in cholesterol (77\%), triglycerides (73\%), and glucose (57\%). ${ }^{15}$ Although most were grade $1 / 2$, the grade $3 / 4$ incidences were $>10 \%$ for the hemoglobin (13\%), lymphocyte (18\%), and glucose (15\%) abnormalities. ${ }^{15}$

Using the safety data from RECORD-1, a multidisciplinary advisory panel has since developed recommendations for managing selected everolimus-associated AEs, as summarized in Table $1 .{ }^{24}$ Given the frequency of hyperglycemia and hyperlipidemia during everolimus therapy, attempts should be made to achieve optimal control following accepted practice guidelines for these conditions prior to initiating treatment with everolimus. Baseline determination and close monitoring of liver function tests is also warranted. In light of the favorable results of RECORD-1, everolimus was granted regulatory approval for treating advanced RCC in VEGFr-TKI-pretreated patients and has since been incorporated into the rapidly evolving US and European clinical practice guidelines (Table 2). 6,25-27

Despite the availability of a growing number of targeted therapies with efficacy (prolongation of PFS and/or OS) in advanced RCC, the use of a single drug generally provides limited benefit, as the disease ultimately progresses due to the development of drug resistance. Resistance is often defined as the cause of tumor progression observed during ongoing therapy based on Response Evaluation Criteria in Solid Tumors (RECIST). However, these criteria may not be the best indicators of resistance to targeted agents. Sensitivity to a targeted therapy is seen when tumors depend on the activity of the target for growth and progression. ${ }^{28}$ In theory, drug resistance can develop through a variety of tumor-related mechanisms, including genetic mutations that change the conformation or structure of a targeted protein, making it inaccessible to drug binding or modifying the interaction of the drug and its target, and adaptive activation of alternative signaling pathways. In addition, susceptibility to targeted therapies may vary between patients because of differences in drug metabolism, including differences in clearance rates that affect drug exposure, tolerability and tumor response.

To extend clinical benefit beyond that of a single therapy, sequential use of targeted therapies has become common practice. Although therapies directed against the same target often have overlapping mechanisms of action, it has long been thought that there is little cross-resistance developing against similar targeted therapies. A survey of seven cancer centers in the United States and Canada 


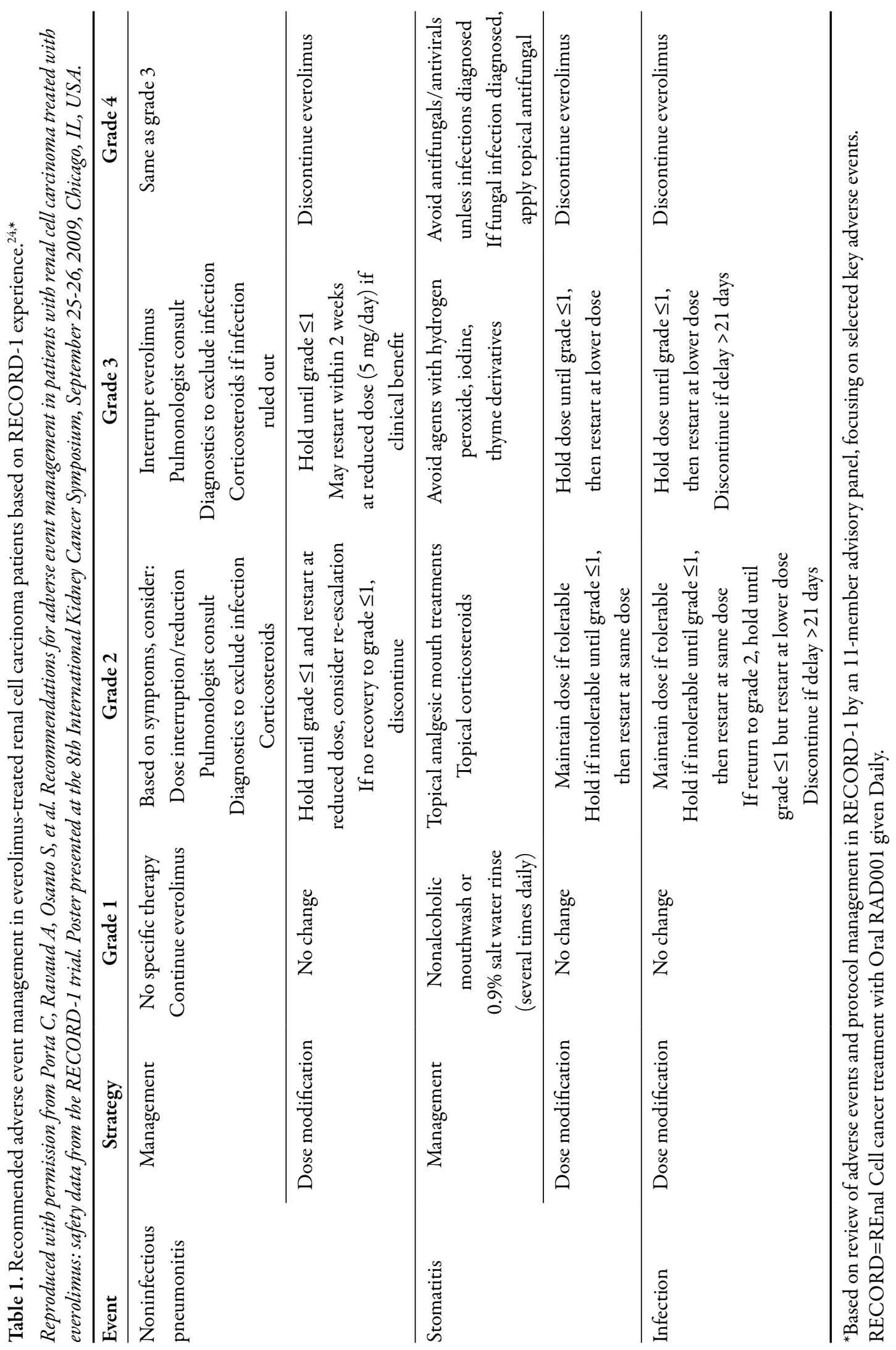




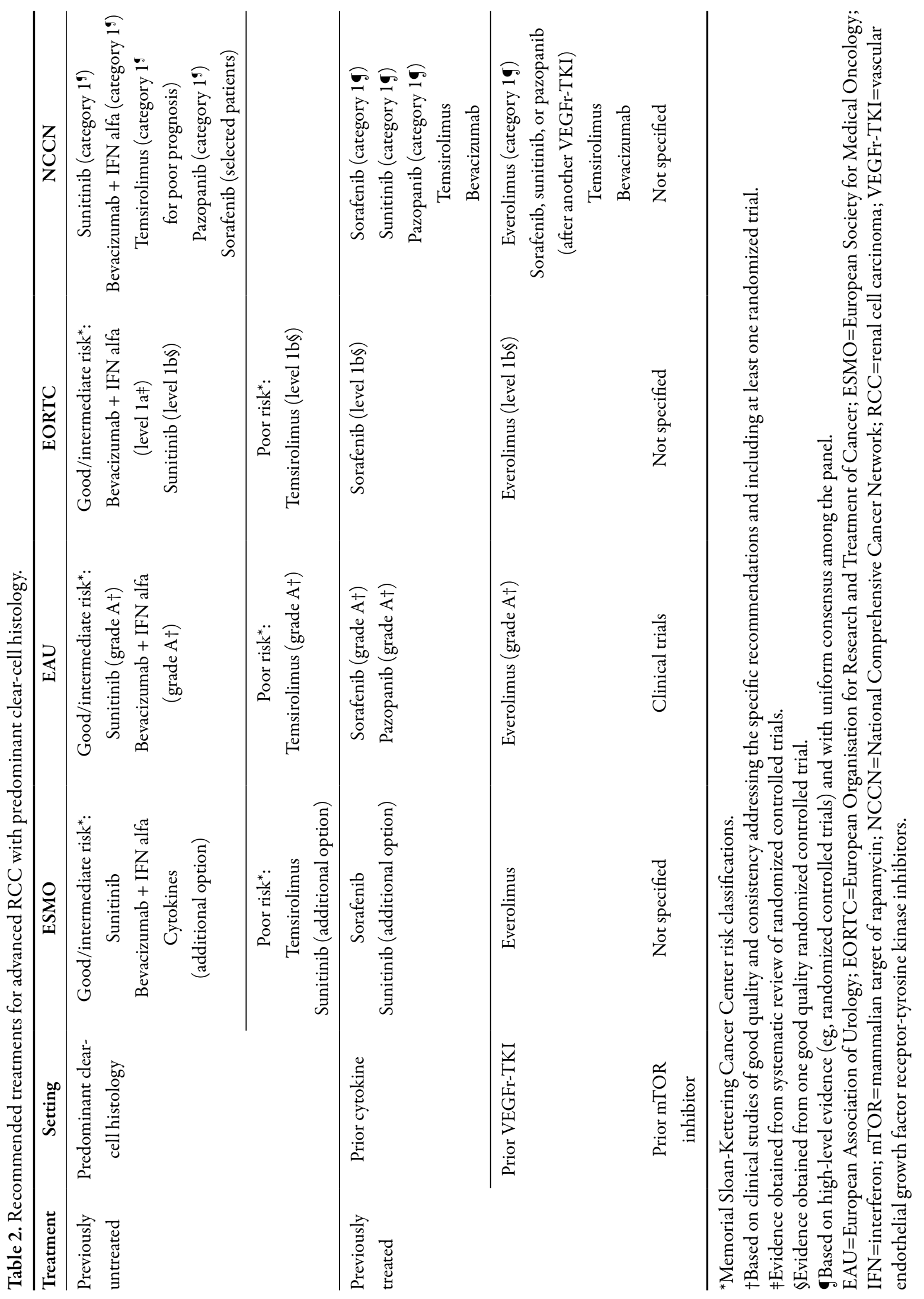


conducted prior to the approval of everolimus as post-TKI therapy found that among patients with metastatic RCC who received first-line VEGF-targeted therapy, 34\% (218/645) received two and $10 \%(70 / 645)$ received three lines of therapy. ${ }^{29}$ Of the 218 patients receiving second-line treatment, 192 received a second VEGF inhibitor (sunitinib, $n=93$; sorafenib, $n=80$; bevacizumab, $n=11$; or axitinib, $n=8$ ). This illustrates the common practice of using sequential therapies against the same or a similar target and is not surprising because until recently TKIs were the only therapeutic options.

Evidence supporting the sequential use of TKIs is limited to relatively small, mainly retrospective, nonrandomized analyses. In two such studies of patients with RCC treated with sorafenib followed by sunitinib or vice versa, the objective response rate ranged from $5 \%$ to $21 \%$ with SD rates from $30 \%$ to $55 \%$ with the second TKI. ${ }^{30,31}$ The variability in efficacy reported for various TKI-TKI regimens may be attributable at least in part to the retrospective nature and small size of the studies and to the differences in strategy employed, such as interval length and reason for change in therapy. To date, only limited data are available from controlled, prospective clinical studies that have compared the efficacy and safety of TKI-TKI sequences in mRCC. In a prospective phase 2 study of sorafenib in sunitinib-refractory patients, the objective response rate was $9.6 \%$, which did not meet the prespecified criteria for a positive study. ${ }^{32}$ In a phase 2 study of sorafenib in patients refractory to bevacizumab or sunitinib, no objective responses were observed. ${ }^{33}$

The results of RECORD-1 demonstrate the effectiveness of one sequential approach in patients with mRCC in a well-designed and wellexecuted prospective randomized trial. Patients who experienced disease progression on or after first-line therapy with a VEGF receptor inhibitor (sunitinib or sorafenib) received significant clinical benefits from everolimus as secondline therapy. ${ }^{14}$ Based on this level I evidence, everolimus has been incorporated into US and European treatment guidelines for RCC with a category 1 /grade A recommendation. ${ }^{6,25-27}$

To further determine the optimum sequence for using sunitinib and everolimus, the RECORD-3 study, a prospective open-label phase 2 crossover study to compare the efficacy and safety of everolimus as first-line therapy followed by second-line sunitinib versus sunitinib as firstline followed by second-line everolimus has been initiated. Ultimately, optimal treatment sequences may need to be patient specific, taking into account comorbid conditions and, if possible, genotypic characteristics.

\section{Ongoing Clinical Trials of Everolimus in RCC}

Everolimus monotherapy is being evaluated further for advanced RCC (Table 3), specifically in patients with the less common non-clearcell subtypes (including the phase 2 RAPTOR study of everolimus in advanced papillary RCC [NCT00688753]), in the neoadjuvant setting, and in sequence with sunitinib. As noted above, in order to determine the optimal sequencing of VEGFr-TKIs and mTOR inhibitors (mTOR inhibitor to TKI or TKI to mTOR inhibitor), the RECORD-3 phase 2 crossover study is randomizing patients with previously untreated metastatic RCC to receive either first-line everolimus followed by sunitinib at time of progressive disease (PD) or first-line sunitinib followed by everolimus upon PD, with PFS as the primary endpoint (NCT00903175). Overall, the majority of everolimus studies that have completed or continue enrolling patients with RCC are investigating dual agent targeted combinations. For example, three separate phase 2 trials are evaluating the combination 
Table 3. Ongoing trials in RCC and NET.*

\begin{tabular}{|c|c|c|c|c|}
\hline & Phase 1 & Phase 1/2 & Phase 2 & Phase 3 \\
\hline $\mathrm{RCC}$ & Everolimus + sunitinib & $\begin{array}{l}\text { Everolimus + sorafenib } \\
\quad \text { (multiple trials) } \\
\text { Everolimus + panobinostat } \\
\text { Everolimus + BNC105P } \\
\text { Everolimus + vatalanib } \\
\text { (various advanced solid } \\
\text { tumors, including RCC) }\end{array}$ & $\begin{array}{l}\text { Monotherapy: non-clear-cell } \\
\text { subtypes (multiple trials, } \\
\text { including RAPTOR) } \\
\text { Monotherapy: neoadjuvant } \\
\text { Monotherapy: vs sunitinib, } \\
\text { first-line and second-line } \\
\text { (RECORD-3) } \\
\text { Everolimus + bevacizumab } \\
\text { (multiple trials, including } \\
\text { RECORD-2) } \\
\text { Everolimus + imatinib } \\
\text { mesylate }\end{array}$ & None ongoing \\
\hline NET & $\begin{array}{l}\text { Everolimus + sorafenib } \\
\text { Everolimus + vatalanib } \\
\text { (various advanced } \\
\text { solid tumors, including } \\
\text { pancreatic NET) } \\
\text { Everolimus + pasireotide }\end{array}$ & Everolimus + temozolomide & $\begin{array}{l}\text { Monotherapy: first- } \\
\text { line for nonfunctioning } \\
\text { gastropancreatic NET } \\
\text { (RAMSETE) } \\
\text { Everolimus + erlotinib }\end{array}$ & $\begin{array}{c}\text { Everolimus }+ \text { octreotide } \\
\text { depot vs octreotide depot } \\
\text { alone in advanced carcinoids } \\
\text { (RADIANT-2) } \\
\text { Everolimus vs placebo in } \\
\text { advanced pancreatic NET } \\
\text { (RADIANT-3) }\end{array}$ \\
\hline
\end{tabular}

${ }^{*}$ Completed accrual (but study ongoing) or active recruitment, per http://clinicaltrials.gov as of February $28,2010$.

NET=neuroendocrine tumors; RCC=renal cell carcinoma.

of everolimus plus bevacizumab, including the RECORD-2 evaluation of everolimus plus bevacizumab versus interferon alfa 2 a plus bevacizumab as first-line treatment of metastatic RCC of clear-cell histology (NCT00719264).

\section{USE OF EVEROLIMUS IN ADVANCED NET}

\section{Overview of the NET Therapeutic Landscape}

NET are relatively rare heterogeneous malignancies thought to originate from neuroendocrine cells scattered throughout the gastrointestinal tract, bronchopulmonary tree, and in various other locations. NET are often metastatic and incurable when diagnosed, and most patients succumb to the disease. An analysis of the Surveillance, Epidemiology, and End Results (SEER) Program registries from 1973 to 2004 determined that median OS in patients with well or moderately differentiated NET with distant metastasis was 33 months. ${ }^{34}$ Surgery represents the only curative option but is suitable only for patients with localized and limited disease. For patients with well and moderately differentiated metastatic NET, treatment decisions have been historically based on the severity of symptoms (stemming from the production of bioactive amines and peptides and/or anatomic location of the tumor), performance status, and the extent of disease burden and progression. ${ }^{4}$ In the past, patients who were relatively asymptomatic or who had slow tumor growth were treated conservatively; 
however, the treatment paradigm has recently shifted with a greater emphasis on disease control. Somatostatin analogs (SAs) are highly effective in symptomatic control of syndromes associated with functional NET and improving quality of life, with recent evidence from the PROMID study supporting true cytostatic potential for octreotide long-acting release (LAR), at least for patients with small intestine (midgut) NET. ${ }^{35}$ Benefit from octreotide LAR was observed in patients with and without symptoms of carcinoid syndrome. ${ }^{35}$ Individual patients may derive benefit from other systemic modalities, such as interferons (for slow-growing intestinal tumors) or cytotoxic chemotherapy (particularly for pancreatic NET), but objective responses and long-term benefits are rare. Radionuclide therapy, with either ${ }^{131} \mathrm{I}-\mathrm{MIBG}$ or a radiolabeled $\left({ }^{90} \mathrm{Y}\right.$ or $\left.{ }^{177} \mathrm{Lu}\right)$ SA, has shown promising results, but long-term prospective studies are lacking and it remains of limited availability.

\section{Preclinical/Mechanistic Rationale for Targeting mTOR}

The rationale for mTOR-targeted therapy for the treatment of advanced NET is based on increased expression/activation of mTOR pathway components and drug efficacy in animal models and cell lines and human tumors. ${ }^{36}$ Expression profiling analyses identified downregulated expression of TSC-2 and PTEN, key inhibitors of the mTOR pathway, in both functioning and nonfunctioning pancreatic NET, and demonstrated that low expression of TSC-2 or PTEN was associated with a worse prognosis. ${ }^{37}$ Moreover, certain genetic syndromes associated with the development of low-grade NET have been found to be related to aberrations of the mTOR pathway; for example, the mutation of $T S C-1 / 2$ gene in tuberous sclerosis, NF-1 in neurofibromatosis, or the $V H L$ gene in VHL disease have been implicated in mTOR activation and the development of NET. ${ }^{38,39}$

The antiproliferative effect of everolimus inhibition on the mTOR pathway has been demonstrated in neuroendocrine cell lines. For example, Zitzmann et al. demonstrated potent dose-dependent inhibition for everolimus on BON human pancreatic NET cells, inducing apoptosis and cell growth arrest. ${ }^{40}$ In a subsequent report describing the mode of action of octreotide and everolimus on the rodent-derived insulin-secreting cell line, ${ }^{3}$ both treatments were shown to inhibit the phosphorylation of p7056K at a site downstream of the serine-threonine kinase Akt and attenuate proliferation. Sensitivity of cells to rapamycin and its derivatives may be limited by a negative feedback loop resulting in increased Akt/mTOR signaling. The addition of octreotide has been shown to sensitize tumor cells to rapamycin treatment by inhibiting Akt activation and increasing the antiproliferative effect of rapamycin in tumor cells. ${ }^{41}$ The inhibitory action of everolimus on cell proliferation and Akt/mTOR/p70S6K pathway activation has been demonstrated in a human medullary thyroid carcinoma (MTC) cell line (TT) and cultured human MTCs. ${ }^{42}$ Everolimus significantly inhibited the cell viability of both TT cells and MTC primary culture cells in a dose-dependent and time-dependent fashion. It inhibited the phosphorylation of Akt downstream targets, mTOR-Ser2448 and p70S6K-Thr389, while Akt phosphorylation was not affected. Moreover, everolimus induced cell cycle arrest in the $\mathrm{G}(0) / G(1)$ phase in TT cells but exerted no effect on cell apoptosis. Overall, the in vitro effects of everolimus on NET cells in these published series and other unpublished experience (available in abstract form $)^{43,44}$ collectively support its clinical evaluation in NET. 


\section{Published Clinical Trials of the Efficacy and Safety of Everolimus in NET}

In 2008, the first phase 2 trial of everolimus in patients with low-grade to intermediate-grade NET was published by Yao et al. ${ }^{45}$ The study enrolled 60 patients with advanced, low-grade to intermediate-grade NET (30 pancreatic NET and 30 lung NET or gastrointestinal NET [carcinoid]). Patients received daily everolimus $5 \mathrm{mg}(n=30)$ or $10 \mathrm{mg}(n=30)$ orally in combination with octreotide LAR (30 mg intramuscularly every 4 weeks). Efficacy was determined by RECIST criteria. The overall response rate was $20 \%$ per intention-to-treat analysis and 23\% in the perprotocol population, in which 13 patients (22\%) achieved a PR, 42 (70\%) maintained SD, and five (22\%) had disease progression. PRs were more frequent in patients with pancreatic NET than in patients with nonpancreatic NET (27\% versus $17 \%)$. Regarding the different dosages, everolimus $10 \mathrm{mg}$ produced a higher PR rate than $5 \mathrm{mg}$ (30\% versus 13\%). Median PFS was 60 weeks overall, longer in patients with nonpancreatic NET (63 weeks) than in patients with pancreatic NET (50 weeks). With regard to tumor shrinkage, the majority of patients showed some degree of tumor reduction by "waterfall" plot. Treatment was generally well tolerated; the most common grade 3/4 AEs were hypophosphatemia (11\%), fatigue $(11 \%)$, diarrhea (11\%), hyperglycemia (9\%), and aphthous ulcers (8\%). ${ }^{45}$ Four patients treated at the $10-\mathrm{mg}$ level developed grade 2 $(n=3)$ or grade $3(n=1)$ pneumonitis, supportively managed with treatment interruption and steroids (and dose reduction to $5 \mathrm{mg}$ for the grade 3 case). ${ }^{45}$ The study demonstrated that everolimus was effective and well tolerated in the treatment of low-grade to intermediategrade NET.

More recently, Yao et al. published the results of an open-label phase 2 study of everolimus in patients with metastatic pancreatic NET after failure of chemotherapy. ${ }^{46}$ In this much larger study, known as RADIANT-1 (RAD001 In Advanced Neuroendocrine Tumors), 160 patients were recruited from 36 centers in 11 countries between June 2006 and June 2007. All participants had confirmed well to moderately differentiated, advanced (unresectable or metastatic) pancreatic NET with RECIST-documented PD during or after chemotherapy. They were divided into two strata by prior octreotide therapy. Patients who had not received octreotide were assigned to stratum 1 (everolimus $10 \mathrm{mg} /$ day, $n=115$ ), while those who were on octreotide LAR for $\geq 3$ consecutive months at study entry were assigned to stratum 2 (everolimus $10 \mathrm{mg} /$ day plus octreotide LAR $30 \mathrm{mg}$ intramuscularly every 4 weeks, $n=45$ ). Efficacy was determined by RECIST at baseline and every 3 months, the biomarkers chromogranin A ( $\mathrm{CgA})$ and neuron-specific enolase (NSE) were evaluated at baseline and monthly if elevated at baseline ${ }^{46}$ and all radiologic images were reviewed by central radiology and investigator assessment. The overall response rate by central radiology was 9.6\% (11/115) in stratum 1 and 4.4\% (2/45) in stratum 2 . In stratum $1, \mathrm{SD}$ was noted in 78 patients $(68 \%)$ while 16 patients $(14 \%)$ had PD. In stratum 2, 36 patients (80\%) had SD and no patient had PD. Efficacy data from both strata were more striking considering that patients in this study had PD upon study entry. Median duration of response by central radiology was 10.6 months in stratum 1 but was not calculated in stratum 2 given the small number of patients. Median PFS was 9.7 months and 16.7 months in strata 1 and 2, respectively. Median OS in stratum 1 was 24.9 months; however, OS had not been reached for stratum 2 at the time of 
data cutoff. The 24-month survival rate was similar in both strata (51\% stratum 1; 55\% stratum 2). An early CgA response (defined as normalization or $\geq 30 \%$ decrease by week 4 ) was seen in $47 \%(33 / 71)$ of patients in stratum 1 and 59\% (13/22) in stratum 2, with early NSE response rates of $72 \%(28 / 39)$ and $50 \%$ (5/10), respectively. ${ }^{46}$ The early biomarker responders also had significantly longer median PFS. Median PFS was 13.3 months in early CgA responders versus 7.5 months in nonearly $\mathrm{CgA}$ responders of stratum 1 , while median PFS in early NSE responders was 8.6 months versus 2.9 months in nonearly responders in stratum 1 . PFS by CgA or NSE in stratum 2 were not evaluated because of the small number of patients.

AEs were generally mild and tolerable, the most common being stomatitis, rash, diarrhea, fatigue, and nausea. The most frequent grade 3/4 AEs were asthenia in stratum 1 (5\%) and thrombocytopenia in stratum 2 (9\%). ${ }^{46}$ Grade $1 / 2$ pneumonitis was reported in both strata $(n=7$ and $n=6$ in strata 1 and 2 , respectively), which was reversible by dosage interruption and modification for the symptomatic grade 2 cases. ${ }^{46}$ Evaluation of the pharmacokinetic effects of coadministration of octreotide LAR and everolimus showed that neither everolimus nor octreotide affect exposure to the other drug. This trial showed promising results in that everolimus, both alone and combined with octreotide LAR, was an effective treatment for advanced pancreatic NET after failure of prior systemic chemotherapy.

\section{Ongoing Clinical Trials of Everolimus in NET}

Clinical trials of everolimus are ongoing across different types of NET (Table 3). Two placebo-controlled phase 3 trials are underway within the RADIANT series, investigating everolimus plus octreotide LAR versus octreotide LAR alone in patients with advanced carcinoids (RADIANT-2, NCT00412061) and everolimus monotherapy for advanced pancreatic NET (RADIANT-3, NCT00363051). 4,36,46 These are the largest randomized controlled trials to be undertaken in this disease setting and are fully enrolled with over 800 patients. Additionally, an openlabel phase 2 study in multiple European centers (RAMSETE, NCT00688623) is exploring everolimus as monotherapy for the first-line treatment of patients with nonfunctioning gastroenteropancreatic NET lacking carcinoid syndrome symptoms. ${ }^{36}$ Additionally, phase 1 and 2 trial designs are combining everolimus with chemotherapy (temozolomide), other targeted agents (bevacizumab, erlotinib, sorafenib, vatalanib), and the investigational SA pasireotide.

\section{NET Case Reports of Interest}

\section{mTOR Inhibition in the Glycemic Control of Insulinoma}

There have been two published case studies on the use of rapamycin and everolimus to control intractable hypoglycemia in patients with insulinoma. ${ }^{47,48}$ Kulke et al. demonstrated the normalization of serum glucose levels after everolimus. ${ }^{47}$ The four patients in their series required depot octreotide, diazoxide, and glucose supplementation for glucose control before commencing everolimus: two achieved PRs while the remaining two had SD after everolimus. All had substantial improvement in glycemic control after receiving everolimus, thereby allowing successful discontinuation of diazoxide and glucose supplements. One patient with tumor regression had recurrent hypoglycemia after stopping 
everolimus. The proposed mechanisms for these very encouraging results were tumor regression plus a direct effect on glycemic control. The latter mechanism is supported by preclinical studies which demonstrate that mTOR inhibition in pancreatic beta cells directly modulates insulin production and release. ${ }^{49,50}$ Consistent with a role of mTOR in glycemic control, hyperglycemia is a common $\mathrm{AE}$ in patients treated with everolimus. ${ }^{15,46}$ A similar effect of rapamycin on treatment of intractable hypoglycemia in insulinoma has been observed. ${ }^{48}$ An older patient with metastatic insulinoma had refractory hypoglycemia while receiving diazoxide, hydrochlorothiazide, octreotide, phenytoin, and dextrose infusion. After starting rapamycin $2 \mathrm{mg} /$ day, glycemic control was maintained solely on rapamycin and hydrochlorothiazide. Interestingly, insulin/glucose ratio showed a steady increase indicating that rapamycin and its derivative everolimus may exert its hyperglycemic effects via increasing peripheral insulin resistance.

\section{Institutional Experience in Malignant Pheochromocytomas and Paragangliomas}

Pheochromocytomas/paragangliomas are NET characteristically resistant to conventional treatment modalities. To date, we have treated four such patients presenting with progressive, metastatic disease after prior treatment with different forms of surgery, chemotherapy, and radionuclide therapy. ${ }^{51}$ All four were treated with everolimus $10 \mathrm{mg} /$ day with or without concomitant chemotherapy (temozolomide and dacarbazine) for 3-6 months until documentation of PD or death. ${ }^{51}$ The results were relatively disappointing, with all patients showing radiological evidence of $\mathrm{PD}$; three died of PD, although one survived $>1$ year after everolimus discontinuation. Everolimus was generally well tolerated, although one patient developed pneumonitis that subsequently improved with conservative treatment. Despite the experience described here, the lack of any treatment with established efficacy for malignant pheochromocytomas supports multicenter and

Table 4. Ongoing phase 3 trials beyond RCC and NET.*

\begin{tabular}{ll}
\hline Tumor type & Phase 3 studies \\
\hline Breast cancer & Neoadjuvant with paclitaxel (GeparQuinto) \\
& HER2-positive locally advanced or metastatic (with paclitaxel and trastuzumab [BOLERO-1] \\
& or vinorelbine and trastuzumab [BOLERO-3]) \\
& With exemestane in estrogen receptor-positive locally advanced or metastatic refractory to \\
& letrozole or anastrozole (BOLERO-2) \\
& Advanced gastric carcinoma (GRANITE-1) \\
Gastrointestinal & Imatinib-resistant gastrointestinal stromal tumors \\
& Advanced hepatocellular carcinoma (EVOLVE-1) \\
Liver & Adjuvant therapy after first-line rituximab chemotherapy in poor-risk diffuse large B-cell \\
Lymphoma & lymphoma (PILLAR-2) \\
& TSC-associated subependymal giant cell astrocytoma (EXIST-1) \\
TSC-associated conditions & TSC-associated or sporadic lymphangioleiomyomatosis-associated angiomyolipoma (EXIST-2) \\
\hline
\end{tabular}

${ }^{*}$ Completed accrual (but study ongoing) or active or pending recruitment, per http://clinicaltrials.gov as of February 28, 2010.

$\mathrm{NET}=$ neuroendocrine tumors; $\mathrm{RCC}=$ renal cell carcinoma; $\mathrm{TSC}=$ tuberous sclerosis complex. 
multinational investigations of this new novel therapy, ideally in combination with additional forms of treatment.

\section{ADDITIONAL PHASE 3 EVEROLIMUS STUDIES AND FUTURE DIRECTIONS}

As summarized in Table 4, numerous phase 3 clinical trials of everolimus are ongoing in tumor types beyond RCC and NET, including those studying combination use with chemotherapy or hormonal therapy for breast cancer, monotherapy for various advanced gastrointestinal and liver malignancies, and adjuvant use for poor-risk diffuse large B-cell lymphoma. Additional phase 3 evaluations of everolimus monotherapy are underway in populations with tuberous sclerosis complex (TSC)-associated subependymal giant cell astrocytoma or TSC-associated or sporadic lymphangioleiomyomatosisassociated angiomyolipoma.

\section{CONCLUSIONS}

In this review we have described the mechanism, efficacy, and safety of everolimus in advanced RCC and various types of advanced NET. Although everolimus does not represent the much needed cure for these rare malignancies, it is undoubtedly effective in improving disease control. The demonstrated benefit of everolimus in VEGFr-TKI-pretreated metastatic RCC has filled a major unmet need, and new questions regarding the optimal sequences and combinations of targeted agents are being addressed in ongoing clinical trials. In addition to reducing the tumor size and controlling disease progression in NET (with or without SA coadministration), emerging evidence suggests that everolimus may improve symptomatic hypoglycemia in malignant insulinomas. Everolimus has displayed an acceptable tolerability profile in clinical trials, with associated AEs mostly of grade $1 / 2$ severity and generally manageable. The results of ongoing phase 3 trials will provide further insight into the role of everolimus as part of combination therapies and as a frontline regimen in the treatment of advanced RCC and NET; additionally, everolimus is being investigated in a number of other solid tumors and hematologic malignancies.

\section{ACKNOWLEDGMENTS}

H-yC has no disclosures. ABG has served as a lecturer and advisory board member for Novartis. RMB has received honoraria from Pfizer, Genentech, Novartis, and Bayer and has served as a consultant for Pfizer, GSK, Novartis, Bayer, Genentech, and Roche.

Editorial assistance in the preparation of this manuscript was provided by Scientific Connexions and funded by Novartis Pharmaceuticals, Inc.

Open Access. This article is distributed under the terms of the Creative Commons Attribution Noncommercial License, which permits any noncommercial use, distribution, and reproduction in any medium, provided the original author(s) and source are credited.

\section{REFERENCES}

1. Sehgal SN, Baker H, Vézina C. Rapamycin (AY-22,989), a new antifungal antibiotic. II. Fermentation, isolation and characterization. J Antibiot (Tokyo). 1975;28:727-732.

2. Douros J, Suffness M. New antitumor substances of natural origin. Cancer Treat Rev. 1981;8:63-87.

3. Grozinsky-Glasberg S, Franchi G, Teng M, et al. Octreotide and the mTOR inhibitor RAD001 (everolimus) block proliferation and interact with the Akt-mTOR-p70S6K pathway in a neuroendocrine tumour cell Line. Neuroendocrinology. 2008;87:168-181. 
4. Chan JA, Kulke MH. Progress in the treatment of neuroendocrine tumors. Curr Oncol Rep. 2009;11:193-199.

5. Vignot S, Faivre S, Aguirre D, Raymond E. mTORtargeted therapy of cancer with rapamycin derivatives. Ann Oncol. 2005;16:525-537.

6. National Comprehensive Cancer Network. NCCN Clinical Practice Guidelines in Oncology ${ }^{\mathrm{TM}}$. V.2.2010. Available at: www.nccn.org. Accessed January 24, 2010.

7. Coppin C, Porzsolt F, Autenrieth M, Kumpf J, Coldman A, Wilt T. Immunotherapy for advanced renal cell cancer. Cochrane Database Syst Rev. 2004;3:CD001425.

8. Motzer RJ, Hutson TE, Tomczak P, et al. Sunitinib versus interferon alfa in metastatic renal-cell carcinoma. N Engl J Med. 2007;356:115-124.

9. Motzer RJ, Hutson TE, Tomczak P, et al. Overall survival and updated results for sunitinib compared with interferon alfa in patients with metastatic renal cell carcinoma. J Clin Oncol. 2009;27:35843590 .

10. Escudier B, Eisen T, Stadler WM, et al. Sorafenib in advanced clear-cell renal-cell carcinoma. N Engl J Med. 2007;356:125-134.

11. Hutson TE, Davis ID, Machiels JP, et al. Efficacy and safety of pazopanib in patients with metastatic renal cell carcinoma. J Clin Oncol. 2010;28:475480 .

12. Escudier B, Pluzanska A, Koralewski $\mathrm{P}$, et al. Bevacizumab plus interferon alfa-2a for treatment of metastatic renal cell carcinoma: a randomised, double-blind phase III trial. Lancet. 2007;370:21032111.

13. Hudes G, Carducci M, Tomczak P, et al. Temsirolimus, interferon alfa, or both for advanced renal-cell carcinoma. N Engl J Med. 2007;356: 2271-2281.

14. Motzer RJ, Escudier B, Oudard S, et al. Efficacy of everolimus in advanced renal cell carcinoma: a double-blind, randomised, placebo-controlled phase III trial. Lancet. 2008;372:449-456.

15. Motzer RJ, Escudier B, Oudard S, et al. Phase 3 trial of everolimus for metastatic renal cell carcinoma: final results and analysis of prognostic factors. Cancer. Published online June 14, 2010.

16. Escudier B, Eisen T, Stadler WM, et al. Sorafenib for treatment of renal cell carcinoma: final efficacy and safety results of the phase III treatment approaches in renal cancer global evaluation trial. J Clin Oncol. 2009;27:3312-3318.

17. Wiederkehr D, Howe CJ, Casciano R, et al. Overall survival among metastatic renal cell carcinoma patients corrected for crossover using inverse probability of censoring weights: analyses from the RECORD-1 phase 3 trial. Eur J Cancer Suppl. $2009 ; 7: 432$.

18. Patel PH, Chadalavada RS, Chaganti RS, Motzer RJ. Targeting von Hippel-Lindau pathway in renal cell carcinoma. Clin Cancer Res. 2006;12:7215-7220.

19. Brugarolas J. Renal-cell carcinoma - molecular pathways and therapies. $\mathrm{N}$ Engl $\mathrm{J}$ Med. 2007;356:185-187.

20. Pantuck AJ, Seligson DB, Klatte $\mathrm{T}$, et al. Prognostic relevance of the mTOR pathway in renal cell carcinoma: implications for molecular patient selection for targeted therapy. Cancer. 2007;109:2257-2267.

21. Robb VA, Karbowniczek M, Klein-Szanto AJ, Henske EP. Activation of the mTOR signaling pathway in renal clear cell carcinoma. J Urol. 2007; 177:346-352.

22. Juengel E, Engler J, Natsheh I, et al. Combining the receptor tyrosine kinase inhibitor AEE788 and the mammalian target of rapamycin (mTOR) inhibitor RAD001 strongly inhibits adhesion and growth of renal cell carcinoma cells. BMC Cancer. 2009;9:161.

23. Amato RJ, Jac J, Giessinger S, Saxena S, Willis JP. A phase 2 study with a daily regimen of the oral mTOR inhibitor RAD001 (everolimus) in patients with metastatic clear cell renal cell cancer. Cancer. 2009;115:2438-2446.

24. Porta C, Ravaud A, Osanto S, et al. Recommendations for adverse event management in patients with renal cell carcinoma treated with everolimus: safety data from the RECORD-1 trial. Poster presented at the 8th International Kidney Cancer Symposium, September 25-26, 2009, Chicago, IL, USA.

25. EAU. Guidelines on Renal Cell Carcinoma. European Association of Urology 2010. Available at: www.uroweb.org. Accessed June 14, 2010.

26. Escudier B, Kataja V; ESMO Guidelines Working Group. Renal cell carcinoma: ESMO clinical recommendations for diagnosis, treatment and follow-up. Ann Oncol. 2009;20(Suppl 4):81-82. 
27. de Reijke TM, Bellmunt J, van Poppel $H$, Marreaud S, Aapro M. EORTC-GU group expert opinion on metastatic renal cell cancer. Eur J Cancer. 2009; 45:765-773.

28. Jänne PA, Gray N, Settleman J. Factors underlying sensitivity of cancers to small-molecule kinase inhibitors. Nat Rev Drug Discov. 2009;8:709-23.

29. Vickers MM, Choueiri TK, Rogers M, et al. Clinical outcome in metastatic renal cell carcinoma patients after failure of initial vascular endothelial growth factor-targeted therapy. J Urol. 2010; March 9 [Epub ahead of print]

30. Sablin MP, Negrier S, Ravaud A, et al. Sequential sorafenib and sunitinib for renal cell carcinoma. J Urol. 2009;182:29-34.

31. Dudek AZ, Zolnierek J, Dham A, Lindgren BR, Szczylik C. Sequential therapy with sorafenib and sunitinib in renal cell carcinoma. Cancer. 2009;115:61-67.

32. Di Lorenzo G, Cartenì G, Autorino R, et al. Phase II study of sorafenib in patients with sunitinibrefractory metastatic renal cell cancer. J Clin Oncol. 2009;27:4469-4474.

33. Shepard DR, Rini BI, Garcia JA, et al. A multicenter prospective trial of sorafenib in patients (pts) with metastatic clear cell renal cell carcinoma (mccRCC) refractory to prior sunitinib or bevacizumab. J Clin Oncol. 2008;26:5123.

34. Yao JC, Hassan M, Phan A, et al. One hundred years after "carcinoid": epidemiology of and prognostic factors for neuroendocrine tumors in 35,825 cases in the United States. J Clin Oncol. 2008;26:30633072 .

35. Rinke A, Müller HH, Schade-Brittinger C, et al. Placebo-controlled, double-blind, prospective, randomized study on the effect of octreotide LAR in the control of tumor growth in patients with metastatic neuroendocrine midgut tumors: a report from the PROMID Study Group. J Clin Oncol. 2009;27:4656-4663.

36. Capurso G, Fazio N, Festa S, et al. Molecular target therapy for gastroenteropancreatic endocrine tumours: biological rationale and clinical perspectives. Crit Rev Oncol Hematol. 2009;72:110124.

37. Missiaglia E, Dalai I, Barbi S, et al. Pancreatic endocrine tumors: expression profiling evidences a role for AKT-mTOR pathway. J Clin Oncol. 2010;28:245-255.
38. Yao JC. Neuroendocrine tumors. Molecular targeted therapy for carcinoid and islet-cell carcinoma. Best Pract Res Clin Endocrinol Metab. 2007;21:163-172.

39. Dworakowska D, Grossman AB. Are neuroendocrine tumours a feature of tuberous sclerosis? A systematic review. Endocr Relat Cancer. 2009;16:45-58.

40. Zitzmann K, De Toni EN, Brand S, et al. The novel mTOR inhibitor RAD001 (everolimus) induces antiproliferative effects in human pancreatic neuroendocrine tumor cells. Neuroendocrinology. 2007;85:54-60.

41. Cerovac V, Monteserin-Garcia J, Rubinfeld H, et al. The somatostatin analogue octreotide confers sensitivity to rapamycin treatment on pituitary tumor cells. Cancer Res. 2010;70:666-674.

42. Grozinsky-Glasberg S, Rubinfeld $\mathrm{H}$, Nordenberg $\mathrm{Y}$, et al. The rapamycin-derivative RAD001 (everolimus) inhibits cell viability and interacts with the Akt-mTOR-p70S6K pathway in human medullary thyroid carcinoma cells. Mol Cell Endocrinol. 2010;315:87-94.

43. Hörsch D, Tielke S, Schrader J. Expression and activation of mTOR in neuroendocrine tumors. Effects of mTOR inhibition by RAD001 upon growth, cell cycle regulation and signalling in neuroendocrine cell lines [abstract]. J Clin Oncol. 2007;25(18S):582s.

44. Righi L, Volante M, Tavaglione V, et al. Mammalian target of rapamycin (MTOR) signaling activation patterns in neuroendocrine tumors of the lung. Virchows Arch. 2008;452(Suppl 1):S15.

45. Yao JC, Phan AT, Chang DZ, et al. Efficacy of RAD001 (everolimus) and octreotide LAR in advanced low- to intermediate-grade neuroendocrine tumors: results of a phase II study. J Clin Oncol. 2008;26:4311-4318.

46. Yao JC, Lombard-Bohas C, Baudin E, et al. Daily oral everolimus activity in patients with metastatic pancreatic neuroendocrine tumors after failure of cytotoxic chemotherapy: a phase II trial. J Clin Oncol. 2010;28:69-76.

47. Kulke $\mathrm{MH}$, Bergsland EK, Yao JC. Glycemic control in patients with insulinoma treated with everolimus. N Engl J Med. 2009;360:195-197.

48. Bourcier ME, Sherrod A, DiGuardo M, Vinik AI. Successful control of intractable hypoglycemia using rapamycin in an 86-year-old man with a pancreatic insulin-secreting islet cell tumor and 
metastases. J Clin Endocrinol Metab. 2009;94:31573162 .

49. Leibiger IB, Leibiger B, Moede T, Berggren PO. Exocytosis of insulin promotes insulin gene transcription via the insulin receptor/PI-3 kinase/ p70 s6 kinase and CaM kinase pathways. Mol Cell. 1998;1:933-938.

50. Fuhrer DK, Kobayashi M, Jiang H. Insulin release and suppression by tacrolimus, rapamycin and cyclosporin A are through regulation of the ATPsensitive potassium channel. Diabetes Obes Metab. 2001;3:393-402.

51. Druce MR, Kaltsas GA, Fraenkel M, Gross DJ, Grossman AB. Novel and evolving therapies in the treatment of malignant phaeochromocytoma: experience with the mTOR inhibitor everolimus (RAD001). Horm Metab Res. 2009;41:697-702. 